02

\title{
Спектроскопическое исследование
} структурных и магнитных свойств $\mathrm{TbCr}_{3}\left(\mathrm{BO}_{3}\right)_{4}$

\author{
(C) Н.Н. Кузьмин ${ }^{1,2,3}$, К.Н. Болдырев ${ }^{1,3}$, В.В. Мальцев ${ }^{2}$ \\ ${ }^{1}$ Институт спектроскопии РАН, \\ 108840 Троицк, Москва, Россия \\ ${ }^{2}$ Московский государственный университет им. М.В. Ломоносова, \\ 119991 Москва, Россия \\ ${ }^{3}$ Московский фризико-технический институт (национальный исследовательский университет), \\ 141700 Долгопрудный, Россия \\ e-mail: kolyanfclm@gmail.com
}

Поступила в редакцию 05.09.2021 г.

В окончательной редакции 15.09.2021 г.

Принята к публикации 22.09.2021 г.

\begin{abstract}
Представлена методика выращивания, изучены структурные особенности и оптические спектры поглощения двойного ортобората $\mathrm{TbCr}_{3}\left(\mathrm{BO}_{3}\right)_{4}$ со структурой хантита. По интенсивностям фононных мод определены соотношения ромбоэдрического и моноклинного политипов у этого соединения в зависимости от условий выращивания. Широкодиапазонные спектры поглощения иона $\mathrm{Tb}^{3+}$ в монокристаллах $\mathrm{TbCr}_{3}\left(\mathrm{BO}_{3}\right)_{4}$ исследованы в интервале температур от комнатной до $3.0 \mathrm{~K}$. По ним определены энергии штарковских уровней

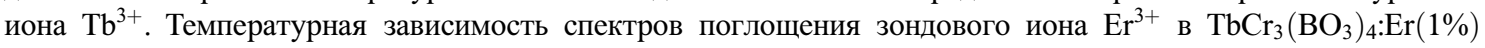
показывает, что имеют место два фазовых перехода и согласуется с предложенной ранее их интерпретацией: при $8.8 \mathrm{~K}$ антиферромагнитно упорядочивается хромовая подсистема, а при $5 \mathrm{~K}$ происходит переориентация магнитных моментов хрома.
\end{abstract}

Ключевые слова: редкоземельные хромовые бораты, магнитное упорядочение, политипия, рост кристаллов, оптическая спектроскопия.

DOI: 10.21883/OS.2022.01.51891.30-21

\section{Введение}

Бораты обладают большим разнообразием структур и хорошо зарекомендовали себя в качестве функциональных материалов [1-3]. Многообразие их кристаллических структур объясняется прежде всего существованием двух типов координационных полиэдров бора $\mathrm{BO}_{3}$-треугольников и $\mathrm{BO}_{4}$-тетраэдров, склонных к полимеризации по различным законам и формированию надструктурных группировок. Бораты применяются в оптических системах [2,4-7], а также как антипирены [8], молекулярные сита [9] и твердые электролиты [10]. С точки зрения коммерческого использования, бораты обладают наибольшим потенциалом для применений в качестве новых оптических материалов, они являются нелинейно-оптическими средами для источников ультрафиолетового (УФ) и глубокого УФ излучения [2,4-6], двулучепреломляющими материалами [11] и активной средой лазеров с самоудвоением и самосмешением частот [12]. Многие бораты имеют нецентросимметричные структуры, широкие окна прозрачности, хорошую химическую стабильность, механическую прочность [2,4-6,13-15]. Кристаллы таких боратов как $\mathrm{KB}_{5} \mathrm{O}_{8} \cdot 4 \mathrm{H}_{2} \mathrm{O}, \mathrm{LiB}_{3} \mathrm{O}_{5}, \beta-\mathrm{BaB}_{2} \mathrm{O}_{4}, \mathrm{CsB}_{3} \mathrm{O}_{5}$, $\alpha-\mathrm{BiB}_{3} \mathrm{O}_{6}, \mathrm{CsLiB}_{6} \mathrm{O}_{10}, \mathrm{KBe}_{2} \mathrm{BO}_{3} \mathrm{~F}_{2}$ и $\mathrm{Sr}_{2} \mathrm{Be}_{2} \mathrm{~B}_{2} \mathrm{O}_{7}$ являются перспективными нелинейно-оптическими материалами $[3,16-22] ; \mathrm{Ca}_{3}\left(\mathrm{BO}_{3}\right)_{2}, \alpha-\mathrm{BaB}_{2} \mathrm{O}_{4}, \mathrm{Ba}_{2} M\left(\mathrm{~B}_{3} \mathrm{O}_{6}\right)_{2}$
$(M=\mathrm{Mg}$ и $\mathrm{Ca}), \mathrm{Ba}_{3} \mathrm{Y}\left(\mathrm{B}_{3} \mathrm{O}_{6}\right)_{2}$ и $\mathrm{Ca}\left(\mathrm{BO}_{2}\right)_{2}$ служат многообещающими двулучепреломляющими материалами для поляризации света в глубоком УФ диапазоне спектра [23-28]. Бораты $\mathrm{YAl}_{3}\left(\mathrm{BO}_{3}\right)_{4}, R \mathrm{MgB}_{5} \mathrm{O}_{10} \quad(R=\mathrm{La}$, $\mathrm{Gd}), R \mathrm{Ca}_{4} \mathrm{O}\left(\mathrm{BO}_{3}\right)_{3} \quad(R=\mathrm{Y}, \mathrm{Gd}), \mathrm{La}_{2} \mathrm{CaB}_{10} \mathrm{O}_{19}$ [29-31], активированные редкоземельными (Р3) ионами, могут быть использованы в качестве активных сред для лазеров.

Значительный интерес представляют двойные бораты, содержащие в своей структуре ионы Р3 элементов и $3 d$-металлов. Это обусловлено тем, что такие соединения могут обладать интересными магнитными свойствами благодаря наличию двух магнитных подсистем [32]. К ним относится обширное семейство соединений хантитоподобных боратов. Известно, что у P3 ферроборатов $R \mathrm{Fe}_{3}\left(\mathrm{BO}_{3}\right)_{4}(R=\mathrm{Y}, \mathrm{La}-\mathrm{Yb})$ магнитная подсистема железа антиферромагнитно упорядочивается при температурах около $40 \mathrm{~K}$ с образованием различных магнитных структур, которые зависят от Р3 иона. В работе [33] было показано, что $\mathrm{GdFe}_{3}\left(\mathrm{BO}_{3}\right)_{4}$ относится к классу мультиферроиков II типа. У $\mathrm{NdFe}_{3}\left(\mathrm{BO}_{3}\right)_{4}$ [34] и $\mathrm{SmFe}_{3}\left(\mathrm{BO}_{3}\right)_{4}$ [35] обнаружен большой магнитоэлектрический эффект. Такие физические свойства делают эти соединения перспективными с точки зрения возможных практических применений.

P3 хромовые бораты $R \mathrm{Cr}_{3}\left(\mathrm{BO}_{3}\right)_{4}(R=\mathrm{La}-\mathrm{Er})$, напротив, мало изучены. В работах $[36,37]$ было показано, что 
для Р3 хромовых боратов характерны две структурные модификации: ромбоэдрическая (пространственная группа (пр.гр.) R32) и моноклинная (пр.гр. C2/c), которые во многих случаях сосуществуют в одном кристалле, а их соотношение зависит от Р3 иона, концентрации исходных компонентов в расплаве и условий роста кристаллов [37,38]. Обе кристаллические структуры состоят из трех сортов координационных полиэдров: тригональных призм $R \mathrm{O}_{6}$, октаэдров $\mathrm{CrO}_{6}$ и треугольников $\mathrm{BO}_{3}$. Октаэдры $\mathrm{CrO}_{6}$ соединяются между собой ребрами и образуют винтовые цепочки вдоль оси $c$; искаженные тригональные призмы ионов Р3 элементов расположены между этими цепочками и изолированы друг от друга. Р3 хромовые бораты с $\mathrm{La}$ [39], $\mathrm{Nd}$ [40], $\mathrm{Sm}$ [41], Eu [42], Gd [43-45], Tb [46], Dy [47] упорядочиваются антиферромагнитно с температурой Нееля $T_{\mathrm{N}}$ в диапазоне температур 6.5-10 K. Имеются немногочисленные работы по синтезу этих соединений $[37,46]$.

В настоящей работе получены спектры поглощения кристаллов тербий-хромового бората в дальней инфракрасной (ИК) обрасти. По этим спектрам обнаружена зависимость соотношения политипных модификаций с пр.гр. $R 32$ и $C 2 / c$ от условий выращивания. Методом эрбиевого спектроскопического зонда определены температуры магнитных фазовых переходов в ромбоэдрическом политипе $\mathrm{TbCr}_{3}\left(\mathrm{BO}_{3}\right)_{4}$. Также в работе представлена схема штарковских уровней энергии иона $\mathrm{Tb}^{3+}$ в $\mathrm{TbCr}_{3}\left(\mathrm{BO}_{3}\right)_{4}$ в парамагнитном состоянии.

\section{Экспериментальная часть}

Выращивание кристаллов тербий-хромового бораma

Кристаллы тербий-хромового бората размером около $0.5 \times 0.5 \times 0.5 \mathrm{~mm}$ выращивались спонтанной кристаллизацией из комплексного раствора-расплава исходя из данных его поля устойчивости в псевдобинарной системе $\mathrm{TbCr}_{3}\left(\mathrm{BO}_{3}\right)_{4}-\mathrm{K}_{2} \mathrm{Mo}_{3} \mathrm{O}_{10}[46]$.

В ростовых экспериментах контроль и регулирование температуры в вертикальных печах с фехралевым нагревателем осуществлялись термоконтроллерами Протерм-100 с комплектами $\mathrm{Pt} / \mathrm{Rh}$-Pt-термопар (градуировка ПП10). Точность поддержания температуры в рабочей зоне печи обычно составляла $\pm 0.1^{\circ} \mathrm{C}$. Содержание растворителя $\left(\mathrm{K}_{2} \mathrm{Mo}_{3} \mathrm{O}_{10}\right)$ изменялось в диапазоне 10-60 wt.\%. Исходные компоненты шихты $\mathrm{Tb}_{4} \mathrm{O}_{7}$, $\mathrm{Cr}_{2} \mathrm{O}_{3}, \mathrm{~B}_{2} \mathrm{O}_{3}, \mathrm{~K}_{2} \mathrm{MoO}_{4}$ и $\mathrm{MoO}_{3}$ (квалификация не ниже „Х.ч.“) помещались в платиновые тигли емкостью $10 \mathrm{ml}$ и нагревались до $1130^{\circ} \mathrm{C}$. Затем в течение $24 \mathrm{~h}$ расплав выдерживался при данной температуре для гомогенизации. Далее происходило его охлаждение со скоростью $1^{\circ} \mathrm{C} / \mathrm{h}$ до $900^{\circ} \mathrm{C}$, после чего его температура быстро понижалась до $300^{\circ} \mathrm{C}$. В конце процесса выращивания тигель извлекался из печи, и кристаллы освобождались от вмещающего их тримолибдата калия растворением его в концентрированной соляной кислоте.
Для исследования магнитных фазовых переходов в $\mathrm{TbCr}_{3}\left(\mathrm{BO}_{3}\right)_{4}$ были получены кристаллы, легированные $1 \%$ Er. Размер таких кристаллов составлял до $0.1 \times 0.1 \times 0.1 \mathrm{~mm}$. Состав исходной шихты в этом эксперименте содержал 50 wt.\% $\mathrm{TbCr}_{3}\left(\mathrm{BO}_{3}\right)_{4}: \operatorname{Er}(1 \%)$ и 50 wt.\% $\mathrm{K}_{2} \mathrm{Mo}_{3} \mathrm{O}_{10}$, поскольку при иных соотношениях шихты и растворителя кристаллы, легированные эрбием, не были получены.

Первичная идентификация выращенных кристаллов осуществлялась на монокристальном дифрактометpe XCalibur S CCD и порошковом дифрактометре ДРОН-3М.

Оптическая спектроскопия тербий-хромового боpama

Спектры поглощения неориентированных кристаллов тербий-хромового бората в ближнем ИК диапазоне, a также порошкового материала $\operatorname{TbCr}_{3}\left(\mathrm{BO}_{3}\right)_{4}: \operatorname{Er}(1 \%)$ в ближнем ИК диапазоне и $\mathrm{TbCr}_{3}\left(\mathrm{BO}_{3}\right)_{4}$ в дальнем и среднем ИК диапазонах были зарегистрированы на фурье-спектрометре Bruker IFS 125HR. Для получения спектров порошкового материала использовалась методика приготовления прессованных таблеток. При измерениях в диапазоне $20-600 \mathrm{~cm}^{-1}$ поликристаллический порошок $\mathrm{TbCr}_{3}\left(\mathrm{BO}_{3}\right)_{4}$ массой $\sim 2 \mathrm{mg}$ перетирался в корундовой ступке $\mathrm{c} \sim 50 \mathrm{mg}$ полиэтилена; при измерениях в диапазонах 400-1700 и 2000-12000 $\mathrm{cm}^{-1}$ образец готовился аналогичным способом из $\sim 5 \mathrm{mg}$ $\mathrm{TbCr}_{3}\left(\mathrm{BO}_{3}\right)_{4}$ и $\sim 40 \mathrm{mg} \mathrm{TbCr}_{3}\left(\mathrm{BO}_{3}\right)_{4}: \mathrm{Er}(1 \%)$ соответственно и $\sim 200 \mathrm{mg} \mathrm{KBr}$. Затем смеси помещались в пресс-форму и спрессовывались в таблетку. Спектры поглощения в дальнем ИК диапазоне были получены при комнатной температуре с разрешением $2 \mathrm{~cm}^{-1}$; в среднем и ближнем ИК диапазонах спектры получены с разрешением до $0.1 \mathrm{~cm}^{-1}$ и в интервале температур $3.0-300 \mathrm{~K}$. Низкотемпературные измерения проводились с использованием криостата замкнутого цикла Sumitomo SRP-082. Контроль температуры осуществлялся контроллером Lakeshore 335 с кремниевым термодиодом, позволяющим измерять температуры в диапазоне $1.8-350 \mathrm{~K}$ с точностью $\pm 0.1 \mathrm{~K}$.

\section{Результаты и обсуждение}

На рис. 1 представлен обзорный фононный спектр поглощения тербий-хромового бората, полученного при составе шихты $50 \mathrm{wt} . \% \mathrm{TbCr}_{3}\left(\mathrm{BO}_{3}\right)_{4}$ и $50 \mathrm{wt} . \%$ $\mathrm{K}_{2} \mathrm{Mo}_{3} \mathrm{O}_{10}$, в диапазоне $50-1650 \mathrm{~cm}^{-1}$ при комнатной температуре. Подобный спектр характерен для всего семейства боратов со структурой хантита $[36,37,46]$.

У Р3 хромовых боратов обнаружены две политипные модификации с пр.гр. $R 32$ и $C 2 / c$. В работе [48] для $\mathrm{GdCr}_{3}\left(\mathrm{BO}_{3}\right)_{4}$ было показано, что основные различия в спектрах поглощения этих модификаций наблюдаются в дальнем ИК диапазоне. Так, моноклинная модификация в отличие от ромбоэдрической имеет полосы поглощения $\sim 73,126,148,248,275 \mathrm{~cm}^{-1}$. В этой же 


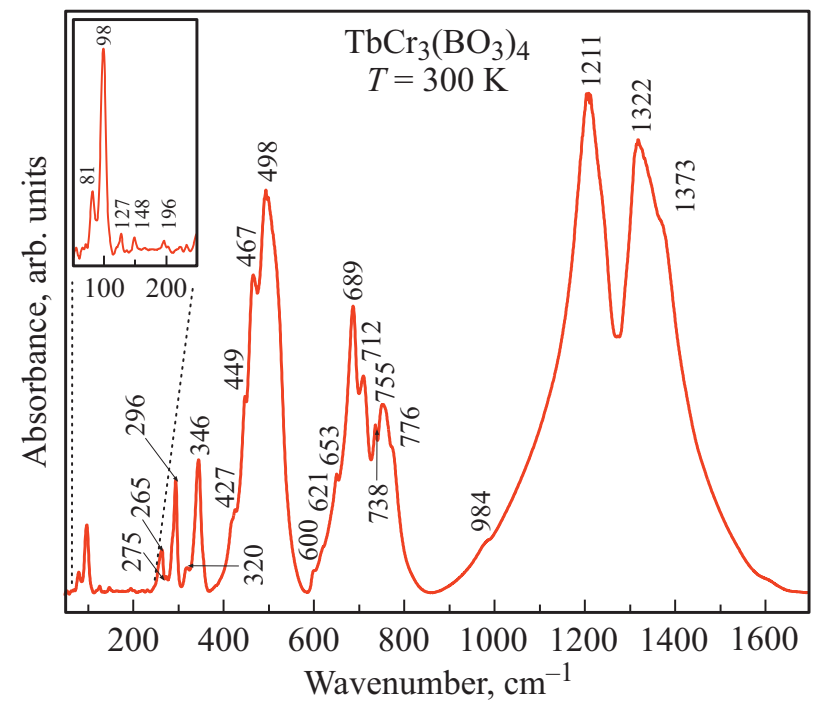

Рис. 1. Обзорный фононный спектр поглощения $\mathrm{TbCr}_{3}\left(\mathrm{BO}_{3}\right)_{4}$ при комнатной температуре.

Таблица 1. Соотношение ромбоэдрического и моноклинного политипов в кристаллах $\mathrm{TbCr}_{3}\left(\mathrm{BO}_{3}\right)_{4}$ при различном соотношении бората и растворителя в шихте

\begin{tabular}{c|c}
\hline $\begin{array}{c}\text { Соотношение бората и } \\
\text { растворителя в шихте }\end{array}$ & $\begin{array}{c}\text { Соотношение модификаций } \\
\text { с пр.гр. } R 32 \text { и } C 2 / c\end{array}$ \\
\hline $40: 60$ & $97: 3$ \\
$50: 50$ & $95: 5$ \\
$60: 40$ & $70: 30$ \\
$70: 30$ & $75: 25$ \\
$80: 20$ & $90: 10$ \\
$90: 10$ & $60: 40$
\end{tabular}

работе описана методика определения их количества по соотношению интенсивностей фононных мод $\sim 73$ и $\sim 79 \mathrm{~cm}^{-1}$ (трансляционные колебания иона $\mathrm{Gd}^{3+}$ в модификациях с пр.гр. $C 2 / c$ и $R 32$ соответственно). Этим модам в $\mathrm{TbCr}_{3}\left(\mathrm{BO}_{3}\right)_{4}$ соответствуют колебания на частотах $\sim 71$ и $\sim 81 \mathrm{~cm}^{-1}$. Согласно этой методике, нами была проведена оценка содержания политипов в выращенных кристаллах. Спектры в дальней ИК области представлены на рис. 2, а результаты определения соотношения политипов - в табл. 1 .

Как следует из табл. 1, при монотонном уменьшении количества растворителя и соответствующем ему пропорциональном увеличением доли кристаллизуемого вещества в растворе-расплаве первоначально возрастает количество более высокотемпературной фазы с пр.гр. $C 2 / c$ [49], что легко объяснимо повышением температуры кристаллизации в этих условиях. Однако после некоторого значения количество фазы с пр. гр. $R 32$ снова возрастает, при этом в таких экспериментах отмечались неполное плавление и слабая раскристаллизация исходной шихты. Вероятнее всего, в данном случае имело место формирование моноклинной модификации $\mathrm{TbCr}_{3}\left(\mathrm{BO}_{3}\right)_{4}$ из небольшого количества растворарасплава, локально образовавшегося в общем объеме непроплавленной шихты, в то время как ромбоэдрическая модификация этого бората синтезировалась при достаточно длительном охлаждении по принципу твердофазной реакции. Сходный с этим механизм фазообразования в многокомпонентных системах наблюдался авторами ранее в работе [50].

В дополнение к фононным спектрам были получены спектры поглощения в диапазоне электронных переходов в ионе $\mathrm{Tb}^{3+}$. На рис. 3 представлен спектр поглощения иона $\mathrm{Tb}^{3+}$ в кристаллах тербий-хромового бората при температуре $10 \mathrm{~K}$. Он состоит из сравнительно узких линий, соответствующих оптическим переходам между энергетическими уровнями в $4 f^{8}$-оболочке иона $\mathrm{Tb}^{3+}$. Этот ион имеет несколько мультиплетов в ИК области, выше $15000 \mathrm{~cm}^{-1}$ в спектрах наблюдаются широкие полосы поглощения, обусловленные ионами $\mathrm{Cr}^{3+}$, что делает затруднительным обнаружение мультиплета ${ }^{5} D_{4}$. Надо отметить, что для исследуемых кристаллов число основных линий в низкотемпературных спектрах поглощения не превышает максимально возможного числа для одного поглощающего центра, что вместе с похожестью на спектры поглощения иона $\mathrm{Tb}^{3+}$ в ферроборате тербия со структурой хантита [51] говорит о значительном преобладании ромбоэдрической модификации над моноклинной.

Кристаллическое поле в зависимости от его симметрии полностью или частично снимает вырождение энергетических уровней свободного иона с целым спином. Так, например, кристаллическое поле симметрии $D_{3}$

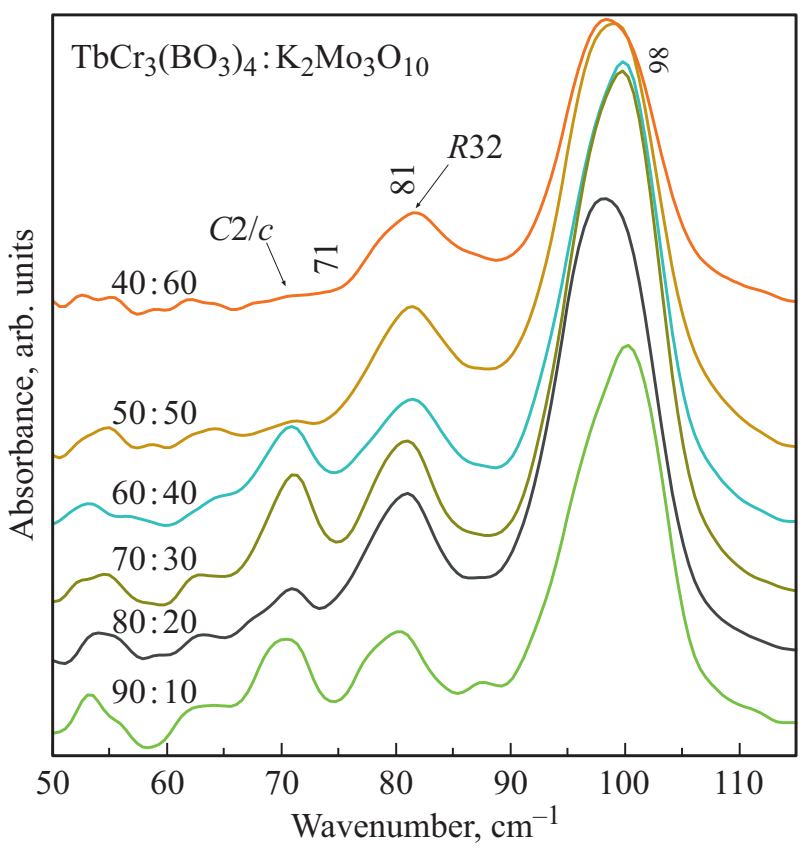

Рис. 2. Спектры поглощения тербий-хромового бората в дальнем ИК диапазоне при различном соотношении бората и растворителя в шихте. 


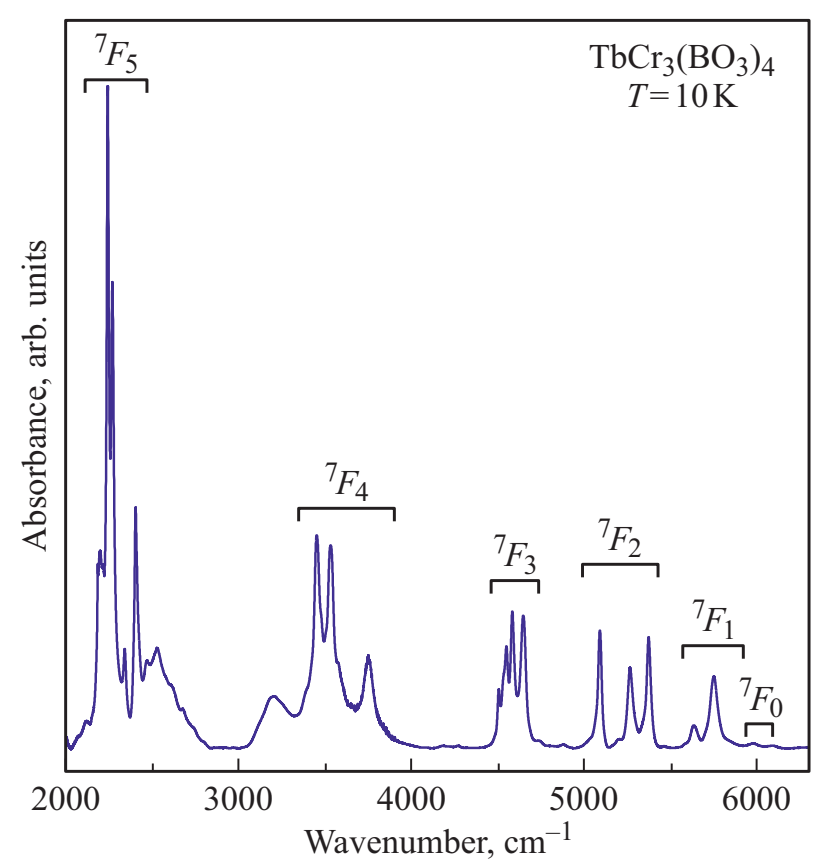

Рис. 3. Спектр поглощения иона $\mathrm{Tb}^{3+}$ в парамагнитном состоянии кристалла $\mathrm{TbCr}_{3}\left(\mathrm{BO}_{3}\right)_{4}$ при температуре $10 \mathrm{~K}$.

Таблица 2. Энергии $(E)$ штарковских уровней $\mathrm{Tb}^{3+}$ в парамагнитном $\mathrm{TbCr}_{3}\left(\mathrm{BO}_{3}\right)_{4}$

\begin{tabular}{l|c}
\hline \multicolumn{1}{c|}{$E, \mathrm{~cm}^{-1}$} & ${ }^{2 S+1} L_{J}$ \\
\hline $0 ; 203 ; 250$ & ${ }^{7} F_{6}$ \\
2183;2196;2213;2240;2268;2339;2402 & ${ }^{7} F_{5}$ \\
3451;3532;3576;3752 & ${ }^{7} F_{4}$ \\
$4505 ; 4532 ; 4549 ; 4583 ; 4647$ & ${ }^{7} F_{3}$ \\
$5091 ; 5265 ; 5373$ & ${ }^{7} F_{2}$ \\
$5635 ; 5751$ & ${ }^{7} F_{1}$ \\
5981 & ${ }^{7} F_{0}$
\end{tabular}

расщепляет уровень ${ }^{7} F_{6}$ свободного иона $\mathrm{Tb}^{3+}$ на девять подуровней. При низких температурах возбужденные уровни основного мультиплета ${ }^{7} F_{6}$ иона тербия не заселены, что позволяет из оптических спектров определить энергии уровней возбужденных мультиплетов в этом ионе. Таким образом, из низкотемпературных спектров поглощения были получены энергии уровней возбужденных мультиплетов $\mathrm{Tb}^{3+}$, а из температурной зависимости спектров поглощения были установлены три энергетических уровня основного мультиплета (рис. $4, a$, табл. 2). Надо отметить, что некоторые спектральные линии имеют асимметричную форму, например все линии мультиплета ${ }^{7} F_{2}$ иона $\mathrm{Tb}^{3+}{ }_{\text {в }} \mathrm{TbCr}_{3}\left(\mathrm{BO}_{3}\right)_{4}: \operatorname{Er}(1 \%)$. При низких температурах контур таких линий превращается в основную линию и спутник (рис. 4, $b$ ). Такие спутники смещены в высокочастотную сторону от основной линии на $8-35 \mathrm{~cm}^{-1}$. Подобные спутники встречались ранее в $\mathrm{TbFe}_{3}\left(\mathrm{BO}_{3}\right)_{4}[51]$, где их появление было связано с оптическими $f-f$-переходами в ионах $\mathrm{Tb}^{3+}$ вблизи дефектов решетки, которыми выступали примеси молибдена и висмута. Эти примеси попадали в кристаллическую структуру из растворителя в процессе роста кристаллов. Большее число спутников у $\mathrm{TbCr}_{3}\left(\mathrm{BO}_{3}\right)_{4}: \operatorname{Er}(1 \%)$ объясняется тем, что при его выращивании в качестве растворителя использовался тримолибдат калия, в котором $\mathrm{MoO}_{3}$ не связан с $\mathrm{Bi}_{2} \mathrm{O}_{3}$ [52], а также имеет значение примесь эрбия (поскольку у $\mathrm{TbCr}_{3}\left(\mathrm{BO}_{3}\right)_{4}$ таких спутников меньше).

Исследование магнитных фазовых переходов в $\mathrm{TbCr}_{3}\left(\mathrm{BO}_{3}\right)_{4}$ было проведено методом эрбиевого спектроскопического зонда. Редкоземельное спектроскопическое зондирование наиболее часто применяется для решения задач структурной химии по определению ближайшего окружения координационного центра [53]. В группе М.Н. Поповой этот же метод (с использованием иона $\mathrm{Er}^{3+}$ в качестве зонда) был использован с целью изучения магнитных фазовых переходов и определения магнитных структур. С его помощью были исследованы такие соединения как $R_{2} \mathrm{Cu}_{2} \mathrm{O}_{5}, \quad R_{2} \mathrm{BaCuO}_{5}, \quad R_{2} \mathrm{BaNiO}_{5}, R \mathrm{Fe}_{3}\left(\mathrm{BO}_{3}\right)_{4} \quad(R=\mathrm{Y}$, $\mathrm{La}-\mathrm{Lu})$ [54-62]. На рис. 5, $a$ представлен спектр поглощения парамагнитного $\mathrm{TbCr}_{3}\left(\mathrm{BO}_{3}\right)_{4}: \operatorname{Er}(1 \%)$ в области перехода ${ }^{4} I_{15 / 2} \rightarrow{ }^{4} I_{13 / 2}$ в ионе $\mathrm{Er}^{3+}$. В кристаллическом поле с симметрией ниже кубической энергетические уровни свободного иона с нечетным числом электронов (каким является ион $\mathrm{Er}^{3+}$ ) расщепляются на крамерсовские дублеты в соответствие с формулой $(2 J+1) / 2$, где $J$ - полный угловой момент электронов. Таким образом, в тербий-хромовом борате, где ион $\mathrm{Er}^{3+}$ занимает позицию с симметрией $D_{3}$, образуется 8 штарковских подуровней для основного мультиплета ${ }^{4} I_{15 / 2}$ и 7 для мультиплета ${ }^{4} I_{13 / 2}$.

В отсутствие магнитного поля дублеты Крамерса остаются двукратно вырожденными, и это вырождение не может быть снято никаким возмущением, кроме магнитного поля. Появление магнитного поля, внешнего или внутреннего (возникающего при магнитном упорядочении кристалла), снимает крамерсовское вырождение, и соответствующая спектральная линия расщепляется максимум на четыре компоненты (см. схему рис. $5, c$ ). Описанное расщепление спектральных линий является индикатором магнитного упорядочения в системе.

В случае тербий-хромового бората магнитные фазовые переходы происходят при температурах ниже $10 \mathrm{~K}$. Рис. $5, b$ показывает, что четыре линии из семи перехода ${ }^{4} I_{15 / 2} \rightarrow{ }^{4} I_{13 / 2}$ в ионе $\mathrm{Er}^{3+}$ в $\mathrm{TbCr}_{3}\left(\mathrm{BO}_{3}\right)_{4}: \operatorname{Er}(1 \%)$ при низких температурах расщепляются на две компоненты. На рис. 6, $a$ представлена линия IA, принадлежащая спектральному переходу ${ }^{4} I_{15 / 2} \rightarrow{ }^{4} I_{13 / 2}$ в ионе $\mathrm{Er}^{3+}$. Эта линия представляет из себя дублет, причем дублетная структура наблюдается и при температурах значительно выше фазовых переходов. По-видимому, дублет связан с присутствием моноклинной модификации $C 2 / c$. Относительные интенсивности линий дублета не дают прямой 

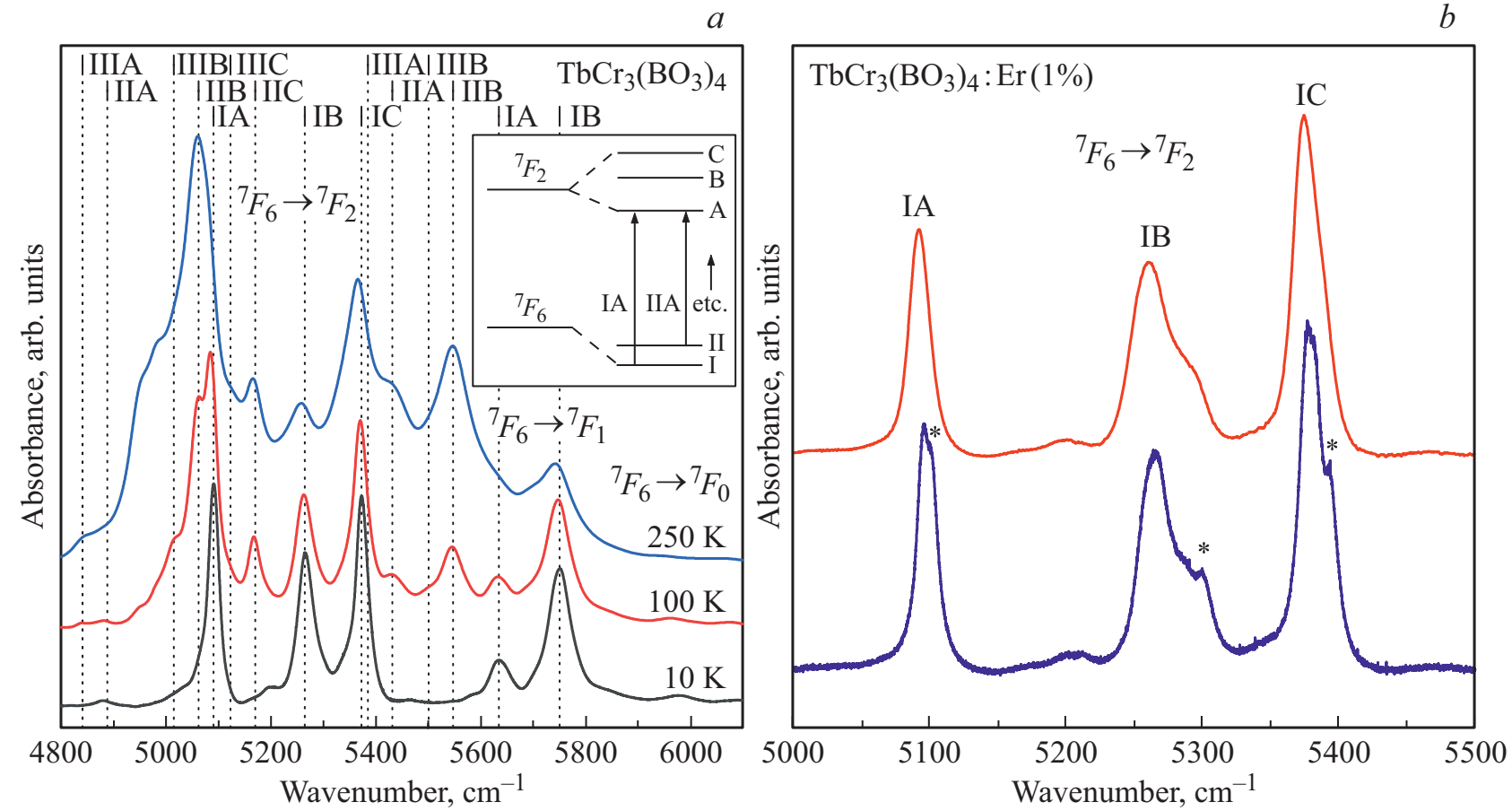

Puc. 4. Спектры поглощения $\mathrm{TbCr}_{3}\left(\mathrm{BO}_{3}\right)_{4}$ в парамагнитном (a), а также $\mathrm{TbCr}_{3}\left(\mathrm{BO}_{3}\right)_{4}: \operatorname{Er}(1 \%)$ в парамагнитном $(10 \mathrm{~K})$ и антиферомагнитном $(3 \mathrm{~K})(b)$ состояниях в области переходов ${ }^{7} F_{6} \rightarrow{ }^{7} F_{2,1,0}$ в ионе $\mathrm{Tb}^{3+}$. На вставке показана схема энергетических уровней иона $\mathrm{Tb}^{3+}$, поясняющая обозначения линий. Звездочкой * обозначены спутники основных линий, обусловленные дефектами решетки.

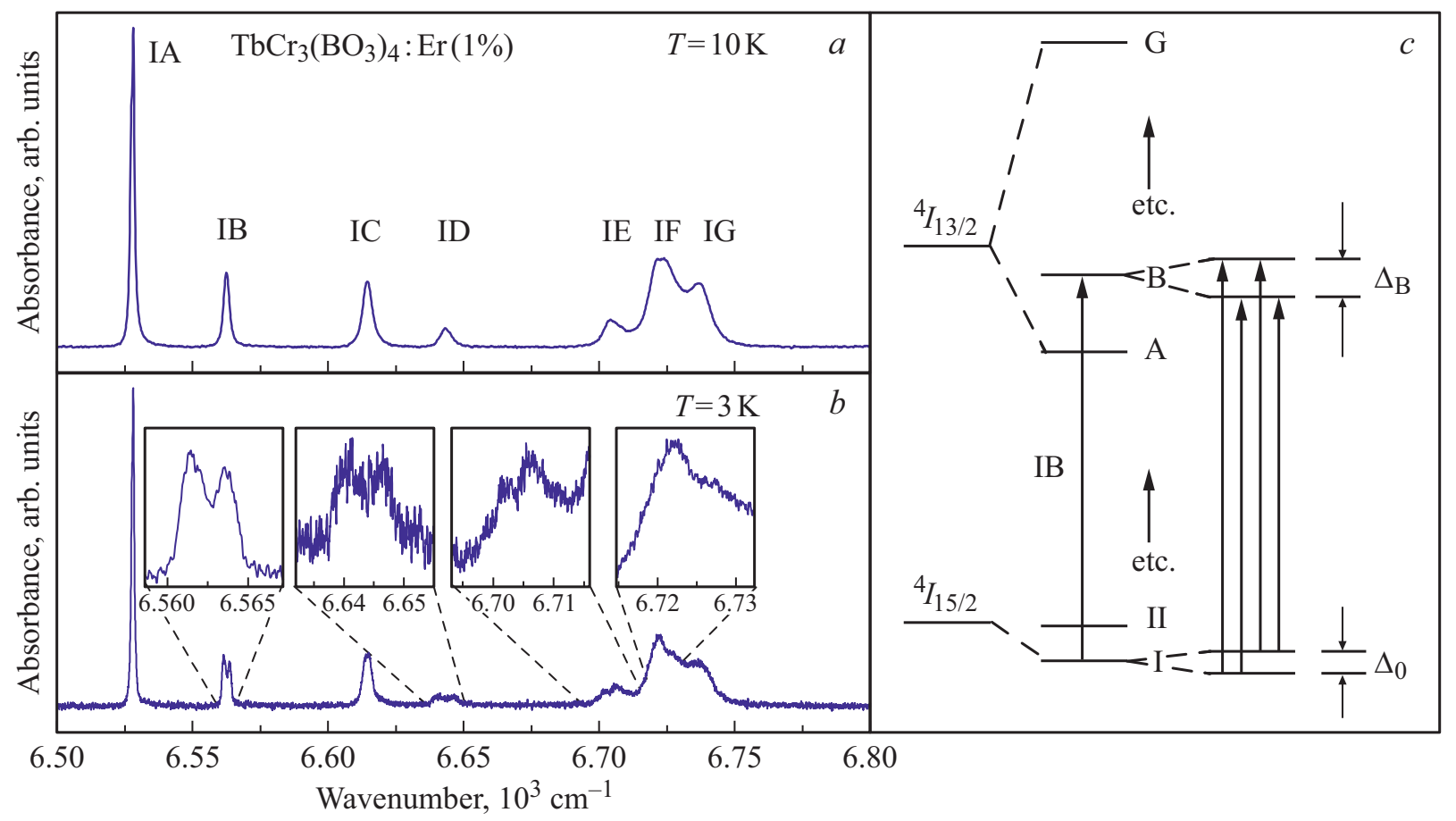

Pис. 5. Спектр поглощения иона $\mathrm{Er}^{3+}$ в парамагнитном $(a)$ и антиферромагнитном $(b) \mathrm{TbCr}_{3}\left(\mathrm{BO}_{3}\right) 4: \operatorname{Er}(1 \%)$; схема энергетических уровней мультиплетов ${ }^{4} I_{15 / 2}$ и ${ }^{4} I_{13 / 2}$ иона $\mathrm{Er}^{3+}(c)$. 

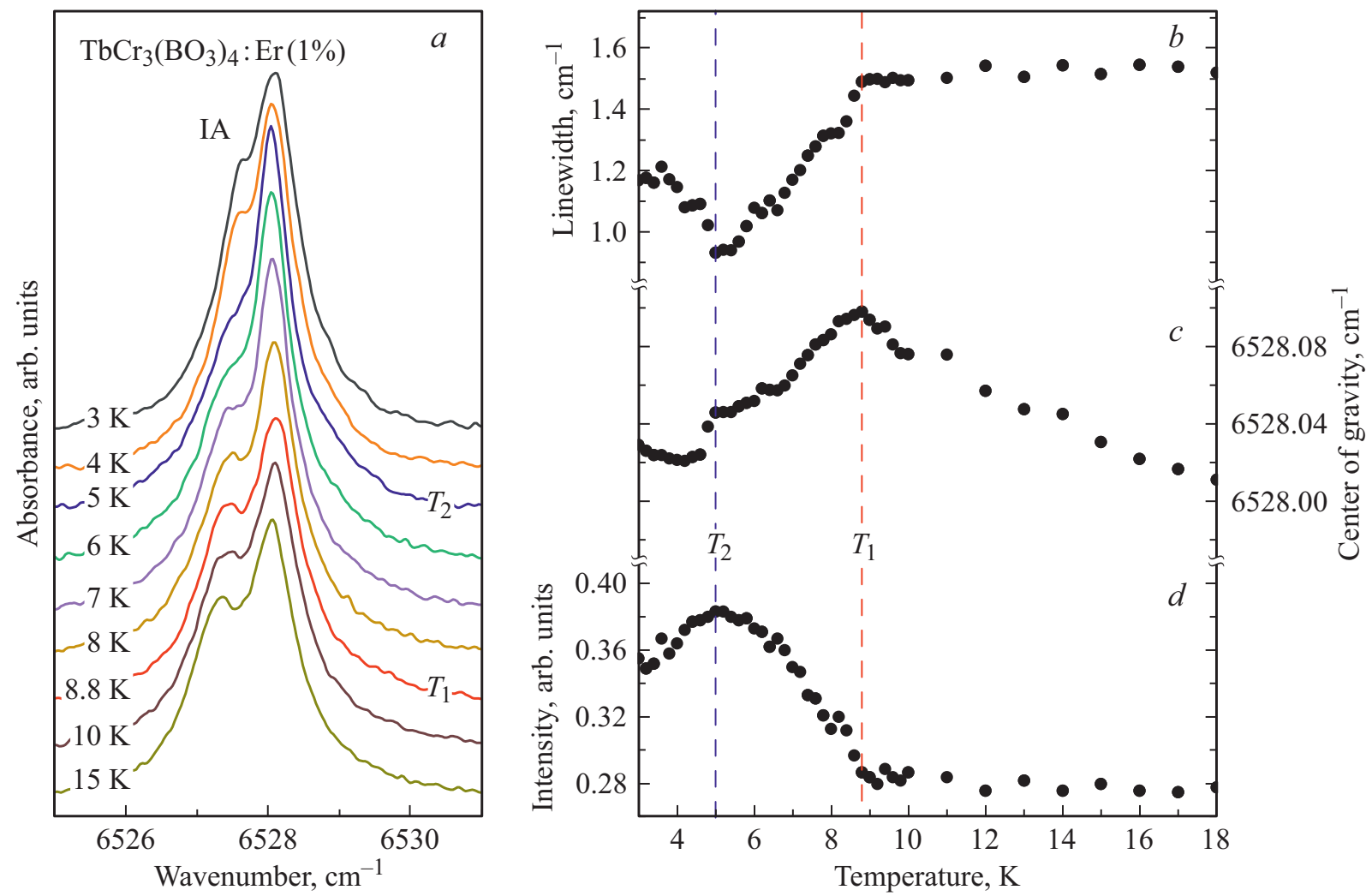

Рис. 6. Линия поглощения IA перехода ${ }^{4} I_{15 / 2} \rightarrow{ }^{4} I_{13 / 2}$ в ионе $\operatorname{Er}^{3+}$ в $\operatorname{TbCr}_{3}\left(\mathrm{BO}_{3}\right)_{4}: \operatorname{Er}(1 \%)$ (a) и температурные зависимости ее полуширины $(b)$, положения центра масс $(c)$, интенсивности $(d)$.

информации об относительном количестве ромбоэдрической и моноклинной модификаций, поскольку силы осцилляторов перехода могут значительно отличаться.

На рис. $6, b-d$ приведены температурные зависимости положения центра масс, полуширины и интенсивности дублетной линии IA. На всех графиках имеются две особенности. При температуре $T_{1}=8.8 \mathrm{~K}$, которая на основании магнитных измерений была сопоставлена с антиферромагнитным упорядочением хромовой подсистемы в $\mathrm{TbCr}_{3}\left(\mathrm{BO}_{3}\right)_{4}$ [46], происходит сужение спектральной линии (что приводит к росту пиковой интенсивности), в то время как дополнительных расщеплений не наблюдается. Подобное поведение наблюдалось, например, в работе [63], где резкое сужение спектральных линий $\mathrm{Sm}^{3+}$ в $\mathrm{P} 3$ францисите $\mathrm{Cu}_{3} \mathrm{Sm}\left(\mathrm{SeO}_{3}\right)_{2} \mathrm{O}_{2} \mathrm{Cl}$ происходило при температуре магнитного упорядочения меди при отсутствии расщепления этих линий. По-видимому, в этих случаях магнитная структура, возникающая в подсистеме $d$-ионов из-за $d-d$-обменного взаимодействия, такова, что $f-d$-обмен (как правило, сильно анизотропный) невелик. Именно $f-d$-обмен определяет расщепление спектральных линий Р3 иона в магнитно-упорядоченном кристалле, содержащем $d$ - и $f$ (Р3)-ионы. В парамагнитной фазе магнитные моменты $d$-ионов флуктуируют по величине и направлению, что приводит к флуктуирующим расщеплениям крамерсовских дублетов Р3 ионов и в результате к уширению спектральных линий [62].
Это уширение снимается при магнитном упорядочении кристалла.

При температуре $T_{2}=5 \mathrm{~K}$ сужение линии IA сменяется уширением. В работе [46] наблюдавшийся при $T_{2}=5 \mathrm{~K}$ фазовый переход был предположительно интерпретирован как спин-переориентационный. Наши данные не противоречат этой интерпретации: наблюдаемое уширение линии IA можно связать с увеличившимся расщеплением крамерсовских дублетов иона $\mathrm{Er}^{3+}$ при изменении ориентации магнитных моментов ионов $\mathrm{Cr}^{3+}$. В явном виде расщепление видно на линиях IB, ID, IE, IF перехода ${ }^{4} I_{15 / 2} \rightarrow{ }^{4} I_{13 / 2}$ в ионе $\mathrm{Er}^{3+}$. С понижением температуры эти линии ведут себя одинаково. Для примера на рис. 7 показана линия поглощения IB, а также температурные зависимости ее положения и величины ее расщепления. Видно, что с контуром линии не происходит изменений до $5 \mathrm{~K}$, после чего линия расщепляется на две компоненты.

Наблюдаемые спектральные зависимости можно объяснить следующим образом. Так как для спектральных линий не обнаружено расщеплений при $T_{1}$, можно заключить, что в диапазоне температур от $T_{1}$ до $T_{2}$ энергетические уровни основного и возбужденного мультиплетов не расщепляются. Поскольку ниже $T_{2}$ спектральные линии либо уширяются, либо имеют две компоненты, относительные интенсивности которых не изменяются с понижением температуры, то это можно 

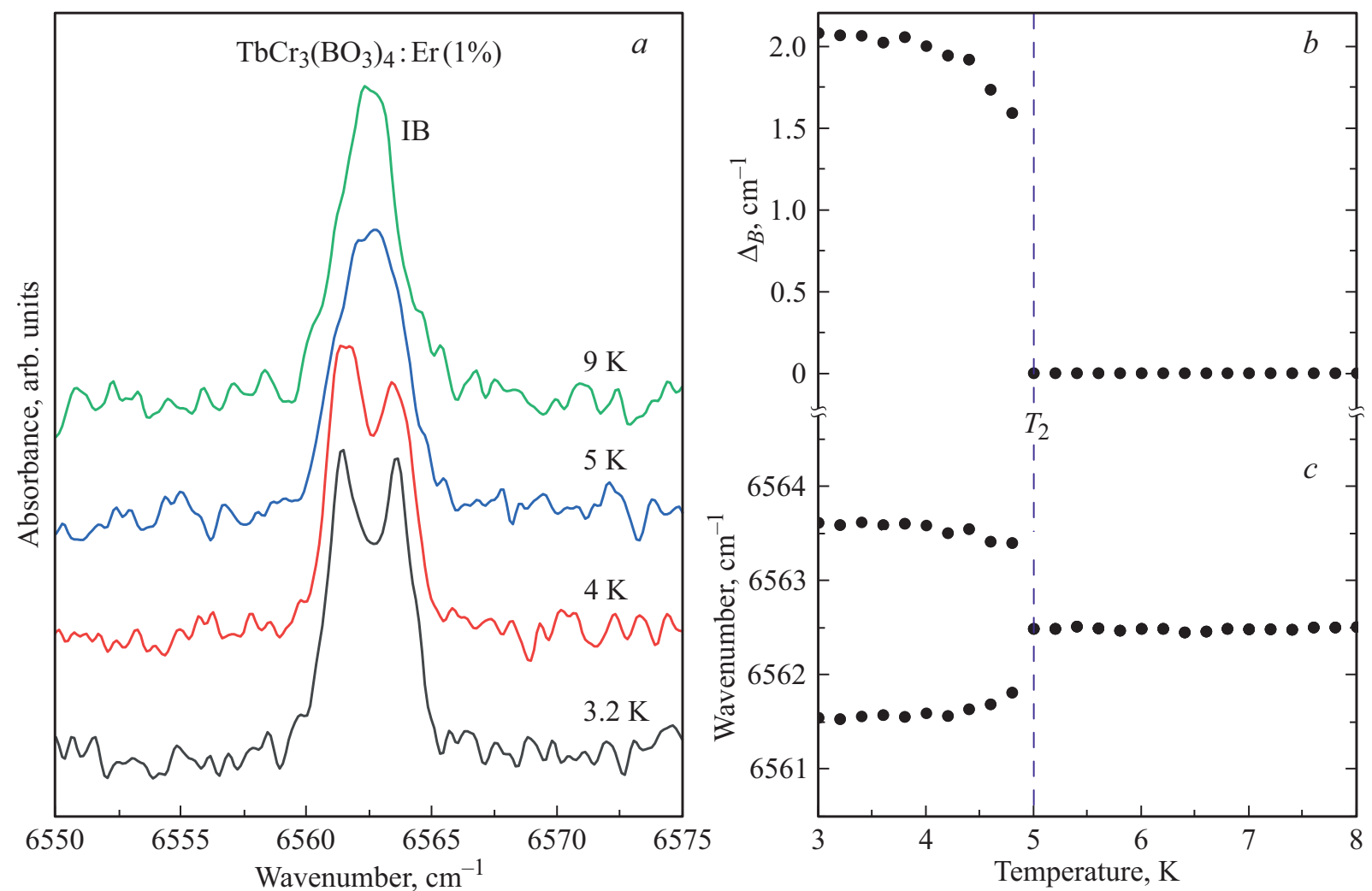

Рис. 7. Низкотемпературные спектры поглощения $\operatorname{TbCr}_{3}\left(\mathrm{BO}_{3}\right) 4: \operatorname{Er}(1 \%)$ в области спектральной линии IB перехода ${ }^{4} I_{15 / 2} \rightarrow{ }^{4} I_{13 / 2}$ в ионе $\mathrm{Er}^{3+}$ в $\operatorname{TbCr}_{3}\left(\mathrm{BO}_{3}\right)_{4}: \operatorname{Er}(1 \%)(a)$ и температурные зависимости величины ее расщепления $(b)$ и положения $(c)$.

связать со специфической схемой расщепления, при которой для основного дублета наблюдается нулевое расщепление (в таком случае две из четырех компонент при магнитном упорядочении совпадают). Таким образом, наблюдаемые расщепления линий соответствуют расщеплениям энергетических уровней возбужденного мультиплета. Для линии IB перехода ${ }^{4} I_{15 / 2} \rightarrow{ }^{4} I_{13 / 2}$ в ионе $\mathrm{Er}^{3+}$ расщепление при $3.0 \mathrm{~K}$ составляет $2.1 \mathrm{~cm}^{-1}$, линии ID $-5.7 \mathrm{~cm}^{-1}$, линии IE $-4.9 \mathrm{~cm}^{-1}$, линии IF $6.7 \mathrm{~cm}^{-1}$.

Расщепление крамерсовских дублетов в приближении среднего поля определяется формулой

$$
\Delta_{k}=\sqrt{\sum\left(g_{k i} B_{i}\right)^{2}}
$$

где $g_{k i}-i$-е $(i=x, y, z)$ компоненты $g$-факторов, $B_{i}-$ магнитное поле, действующее на $k$-й дублет Крамерса. Можно предположить что в интервале температур $T_{1}>T>T_{2}$ анизотропный $g_{k i}$-фактор имеет близкую к нулю $i$-компоненту вдоль магнитного поля $B_{i}$, а при температуре ниже $T_{2}$ она уже имеет не нулевое значение. Поскольку на данных момент Р3 хромовые бораты мало изучены, то для выяснения природы перехода $T_{2}$ необходимы дополнительные исследования.

По сравнению с ферроборатом $\mathrm{TbFe}_{3}\left(\mathrm{BO}_{3}\right)_{4}$ тербий-хромовый борат упорядочивается анитиферромагнитно при более низкой температуре ( $8.8 \mathrm{~K}$ против
$41 \mathrm{~K}$ ), имеет меньшие значения расщеплений $\Delta_{\mathrm{B}}$ и $\Delta_{0}$ для перехода ${ }^{4} I_{15 / 2} \rightarrow{ }^{4} I_{13 / 2}$ в ионе $\mathrm{Er}^{3+}-2.1 \mathrm{~cm}^{-1}$ при $3.0 \mathrm{~K}$ против $5.0 \mathrm{~cm}^{-1}$ при $4.5 \mathrm{~K}$ и $0 \mathrm{~cm}^{-1}$ при $3.0 \mathrm{~K}$ против $1.9 \mathrm{~cm}^{-1}$ при $4.5 \mathrm{~K}$ соответственно [61]. Данный факт свидетельствует о меньшей величине эффективного магнитного поля в $\operatorname{TbCr}_{3}\left(\mathrm{BO}_{3}\right)_{4}$ по сравнению c $\mathrm{TbFe}_{3}\left(\mathrm{BO}_{3}\right)_{4}$.

\section{Заключение}

В работе представлены исследования условий выращивания, структурных особенностей, оптических характеристик тербий-хромового бората со структурой хантита. По соотношению интенсивностей фононных мод $\sim 71$ и $\sim 81 \mathrm{~cm}^{-1}$ проведена оценка содержания политипных модификаций с пр.гр. $R 32$ и $C 2 / c$, что позволит в дальнейшем улучшить методику роста кристаллов $\mathrm{TbCr}_{3}\left(\mathrm{BO}_{3}\right)_{4}$ и получать однофазные соединения. Из температурно-зависимых спектров поглощения тербий-хромового бората в диапазоне электронных переходов в ионе $\mathrm{Tb}^{3+}$ определены энергии штарковских уровней этого иона в модификации c пр.гр. R32. Температурная зависимость спектров поглощения $\mathrm{TbCr}_{3}\left(\mathrm{BO}_{3}\right)_{4}: \operatorname{Er}(1 \%)$ в области перехода ${ }^{4} I_{15 / 2} \rightarrow{ }^{4} I_{13 / 2}$ в зондовом ионе $\mathrm{Er}^{3+}$ подтвердила наличие двух магнитных переходов в тербий-хромовом борате, ранее установленных по магнитным измерени- 
ям [46]. Спектроскопические данные согласуются с предложенной в работе [46] интерпретацией фазовых переходов: при $T_{1}=8.8 \mathrm{~K}$ антиферромагнитно упорядочивается хромовая подсистема в $\mathrm{TbCr}_{3}\left(\mathrm{BO}_{3}\right)_{4}$, а при $T_{2}=5 \mathrm{~K}$ происходит переориентация магнитных моментов хрома.

\section{Благодарности}

Авторы признательны М.Н. Поповой и С.А. Климину за замечания по работе.

\section{Финансирование работы}

Экспериментальные работы по выращиванию кристаллов тербий-хромового бората осуществлялись за счет средств Российского научного фонда (проект № 19-12-00235), спектроскопические исследования выполнялись при поддержке гранта РНФ № 19-12-00413.

\section{Конфликт интересов}

Авторы заявляют, что у них нет конфликтов интереcoB.

\section{Список литературы}

[1] S. Trofimenko. Chemical Reviews, 93 (3), 943 (1993). DOI: $10.1021 / \mathrm{cr} 00019 \mathrm{a} 006$

[2] P. Becker. Advanced Materials, 10 (13), 979 (1998). DOI: $10.1002 /($ SICI $) 1521-$ 4095(199809)10:13<979::AID-ADMA979>3.0.CO;2-N

[3] C. Chen, Y. Wang, B. Wu, K. Wu, W. Zeng, L. Yu. Nature, 373, 322 (1995). DOI: 10.1038/373322a0

[4] C. Chen, G. Liu. Ann. Rev. Materials Science, 16, 203 (1986). DOI: 10.1146/annurev.ms.16.080186.001223

[5] M. Mutailipu, M. Zhang, Z. Yang, S. Pan. Accounts Chem. Research, 52 (3), 791 (2019). DOI: 10.1021/acs.accounts.8b00649

[6] T. Sasaki, Y. Mori, M. Yoshimura, Y.K. Yap, T. Kamimura. Science and Engineering: R: Reports, 30 (1-2), 1 (2000). DOI: 10.1016/S0927-796X(00)00025-5

[7] H. Huang, Y. He, Z. Lin, L. Kang, Y. Zhang. J. Phys. Chem. C, 117 (44), 22986 (2013). DOI: 10.1021/jp4084184

[8] K.K. Shen, S. Kochesfahani, F. Jouffret. Polymers for Advanced Technologies, $19(6), 469$ (2008). DOI: $10.1002 /$ pat.1119

[9] R. Millini, G. Perego, G. Bellussi. Topics in Catalysis, 9 (1), 13 (1999). DOI: 10.1023/A:1019198119365

[10] J.L.C. Rowsell, J. Gaubicher, L.F. Nazar. J. Power Sources, 97-98, 254 (2001). DOI: 10.1016/S0378-7753(01)00532-8

[11] F. Qin, R.K. Li. J. Crystal Growth, 318(1), 642 (2011). DOI: 10.1016/j.jcrysgro.2010.08.037

[12] G. Aka, A. Brenier. Opt. Materials, $22(2), 89$ (2003). DOI: 10.1016/S0925-3467(02)00351-8

[13] S. Wang, E.V. Alekseev, J. Ling, G. Liu, W. Depmeier, T.E. Albrecht-Schmitt. Chemistry of Materials, 22 (6), 2155 (2010). DOI: $10.1021 / \mathrm{cm} 9037796$

[14] E.R. Wang, J.H. Huang, S.J. Yu, Y.Z. Lan, J.W. Cheng, G.Y. Yang. Inorganic Chemistry, 56(12), 6780 (2017). DOI: 10.1021/acs.inorgchem.7b00975
[15] C. Chen, Y. Wu, R. Li. Intern. Rev. Phys. Chem., 8(1), 65 (1989). DOI: 10.1080/01442358909353223

[16] C.F. Dewey, W.R. Cook, R.T. Hodgson, J.J. Wynne. Appl. Phys. Lett., 26 (12), 714 (1975). DOI: 10.1063/1.88047

[17] C. Chen, B. Wu, A. Jiang, G. You. Science in China, Series B - Chemistry, Biological, Agricultural, Medical \& Earth Sciences, 28(3), 235 (1985). DOI: $10.1360 / y b 1985-28-3-235$

[18] C. Chen, Y. Wu, A. Jiang, B. Wu, G. You, R. Li, S. Lin. J. Opt. Soc. Am. B, 6(4), 616 (1989). DOI: $10.1364 / \mathrm{JOSAB} .6 .000616$

[19] Y. Wu, T. Sasaki, N. Nakai, A. Yokotani, H. Tang, C. Chen. Appl. Phys. Lett., 62 (21), 2614 (1993). DOI: $10.1063 / 1.109262$

[20] Y. Mori, I. Kuroda, S. Nakajima, T. Sasaki, S. Nakai. Appl. Phys. Lett., 67 (13), 1818 (1995). DOI: 10.1063/1.115413

[21] H. Hellwig, J. Liebertz, L. Bohatý. Solid State Commun., 109 (4), 249 (1998). DOI: 10.1016/S0038-1098(98)00538-9

[22] C.T. Chen, G.L. Wang, X.Y. Wang, Z.Y. Xu. Appl. Phys. B, 97, 9 (2009). DOI: 10.1007/s00340-009-3554-4

[23] Z. Guoqing, X. Jun, C. Xingda, Z. Heyu, W. Siting, X. Ke, D. Peizhen, G. Fuxi. J. Crystal Growth, 191 (3), 517 (1998). DOI: $10.1016 / \mathrm{S} 0022-0248(98) 00162-6$

[24] S. Zhang, X. Wu, Y. Song, D. Ni, B. Hu, T. Zhou. J. Crystal Growth, $252(1-3), 246$ (2003). DOI: 10.1016/S0022-0248(03)00867-4

[25] M. He, X. Chen, Y. Sun, J. Liu, J. Zhao, C. Duan. Crystal Growth \& Design, 7(2), 199 (2007). DOI: $10.1021 / \operatorname{cg} 0606141$

[26] Z. Jia, N. Zhang, Y. Ma, L. Zhao, M. Xia, R. Li. Crystal Growth \& Design, $17(2), \quad 558 \quad$ (2017). DOI: 10.1021/acs.cgd.6b01428

[27] R.K. Li, Y.Y. Ma. CrystEngComm, 14, 5421 (2012). DOI: $10.1039 / \mathrm{C} 2 \mathrm{CE} 25240 \mathrm{~F}$

[28] X. Chen, B. Zhang, F. Zhang, Y. Wang, M. Zhang, Z. Yang, K.R. Poeppelmeier, S. Pan. J. Am. Chem. Society, 140 (47), 1631 (2018). DOI: $10.1021 /$ jacs.8b10009

[29] Y. Huang, H. Chen, S. Sun, F. Yuan, L. Zhang, Z. Lin, G. Zhang, G. Wang. J. Alloys and Compounds, 646, 1083 (2015). DOI: 10.1016/j.jallcom.2015.06.196

[30] P. Dekker, J.M. Dawes, J.A. Piper, Y. Liu, J. Wang. Opt. Commun., 195(5-6), 431 (2001). DOI: $10.1016 / \mathrm{S} 0030-4018(01) 01347-5$

[31] B. Denker, B. Galagan, L. Ivleva, V. Osiko, S. Sverchkov, I. Voronina, J.E. Hellstrom, G. Karlsson, F. Laurell. Appl. Phys. B, 79 (5), 577 (2004). DOI 10.1007/s00340-004-1605-4

[32] А.М. Кадомцева, Ю.Ф. Попов, Г.П. Воробьев, А.П. Пятаков, С.С. Кротов, К.И. Камилов, В.Ю. Иванов, А.А. Мухин, А.К. Звездин, А.М. Кузьменко, Л.Н. Безматерных, И.А. Гудим, В.Л. Темеров. Физика низких температур, 36 (6), 640 (2010). [A.M. Kadomtseva, Yu.F. Popov, G.P. Vorob'ev, A.P. Pyatakov, S.S. Krotov, K.I. Kamilov, V.Yu. Ivanov, A.A. Mukhin, A.K. Zvezdin, A.M. Kuz'menko, L.N. Bezmaternykh, I.A. Gudim, V.L. Temerov. Low Temperature Physics, 36 (6), 511 (2010). DOI: $10.1063 / 1.3457390]$

[33] А.К. Звездин, С.С. Кротов, А.М. Кадомцева, Г.П. Воробьев, Ю.Ф. Попов, А.П. Пятаков, Л.Н. Безматерных, Е.А. Попова. Письма в Журн. эксперимент. и теор. физики, 81 (6), 335 (2005). [A.K. Zvezdin, S.S. Krotov, A.M. Kadomtseva, G.P. Vorob'ev, Y.F. Popov, A.P. Pyatakov, L.N. Bezmaternykh, 
E.A. Popova. J. Experiment. and Theor. Phys. Lett., 81 (6), 272 (2005). DOI: 10.1134/1.1931014]

[34] А.К. Звездин, С.С. Кротов, А.М. Кадомцева, Г.П. Воробьев, Ю.Ф. Попов, А.П. Пятаков, Л.Н. Безматерных, А.В. Кувардин, Е.А. Попова. Письма в Журн. эксперимент. и теор. физики, $83(11), 509$ (2006). [A.K. Zvezdin, G.P. Vorob'ev, A.M. Kadomtseva, Y.F. Popov, A.P. Pyatakov, L.N. Bezmaternykh, A.V. Kuvardin, E.A. Popova. J. Experiment. and Theor. Phys. Lett., 83 (11), 509 (2006). DOI: 10.1134/S0021364006110099]

[35] Ю.Ф. Попов, А.П. Пятаков, А.М. Кадомцева, Г.П. Воробьев, А.К. Звездин, А.А. Мухин, В.Ю. Иванов, И.А. Гудим. ЖЭТФ, $138(2), 226$ (2010). [Y.F. Popov, A.P. Pyatakov, A.M. Kadomtseva, G.P. Vorob'ev, A.K. Zvezdin, A.A. Mukhin, V.Yu. Ivanov, I.A. Gudim. J. Experiment. and Theor. Phys., 111 (2), 199 (2010). DOI: $10.1134 / \mathrm{S} 1063776110080066]$

[36] В.С. Куражковская, Е.А. Добрецова, Е.Ю. Боровикова, В.В. Мальцев, Н.И. Леонюк. Журнал структурной химии, 52 (4), 721 (2011). [V.S. Kurazhkovskaya, E.A. Dobretsova, E.Y. Borovikova, V.V. Mal'tsev, N.I. Leonyuk. J. Structural Chemistry, 52 (4), 699 (2011). DOI: $10.1134 / \mathrm{S} 0022476611040081]$

[37] E.Y. Borovikova, E.A. Dobretsova, K.N. Boldyrev, V.S. Kurazhkovskaya, V.V. Maltsev, N.I. Leonyuk. Vibrational Spectroscopy, $\quad \mathbf{6 8}, \quad 82 \quad$ (2013). DOI: $10.1016 /$ j.vibspec.2013.05.004

[38] К.Н. Болдырев, Н.Н. Кузьмин, Е.А. Добрецова. Опт. и спектроск., $\mathbf{1 2 9}(1), 41 \quad$ (2021). DOI: 10.21883/OS.2021.01.50437.248-20 [K.N. Boldyrev, N.N. Kuzmin, E.A. Dobretsova. Opt. Spectrosc., 129 (1), 37 (2021). DOI: 10.1134/S0030400X21010033]

[39] А.Н. Блудов, Ю.А. Савина, В.А. Пащенко, С.Л. Гнатченко, И.В. Колодий, В.В. Мальцев, Н.Н. Кузьмин, Н.И. Леонюк. Физика низких температур, 46 (6), 767 (2020). [A.N. Bludov, Y.O. Savina, V.A. Pashchenko, S.L. Gnatchenko, I.V. Kolodiy, V.V. Mal'tsev, N.N. Kuzmin, N.I. Leonyuk. Low Temperature Physics, 46 (6), 643 (2020). DOI: $10.1063 / 10.0001250]$

[40] E.A. Popova, N.I. Leonyuk, M.N. Popova, E.P. Chukalina, K.N. Boldyrev, N. Tristan, R. Klingeler, B. Büchner. Phys. Rev. B, 76 (5), 054446 (2007). DOI: 10.1103/PhysRevB.76.054446

[41] К.Н. Болдырев, Е.П. Чукалина, Н.И. Леонюк. ФТТ, 50 (9), 1617 (2008). [K.N. Boldyrev, E.P. Chukalina, N.I. Leonyuk. Phys. Solid State, 50(9), 1681 (2008). DOI: $10.1134 / \mathrm{S} 1063783408090187]$

[42] Ł. Gondek, A. Szytuła, J. Przewoźnik, J. Żukrowski, A. Prokhorov, L. Chernush, E. Zubov, V. Dyakonov, R. Duraj, Yu. Tyvanchuk. J. Solid State Chemistry, 210 (1), 30 (2014). DOI: 10.1016/j.jssc.2013.10.029

[43] А.Н. Блудов, Ю.А. Савина, В.А. Пащенко, С.Л. Гнатченко, В.В. Мальцев, Н.Н. Кузьмин, Н.И. Леонюк. Физика низких температур, 44 (5), 554 (2018). [A.N. Bludov, Y.O. Savina, V.A. Pashchenko, S.L. Gnatchenko, V.V. Mal'tsev, N.N. Kuzmin, N.I. Leonyuk. Low Temperature Physics, 44 (5), 423 (2018). DOI: 10.1063/1.5034153]

[44] А.Н. Блудов, Ю.А. Савина, М.И. Кобец, В.А. Пащенко, С.Л. Гнатченко, Н.Н. Кузьмин, В.В. Мальцев, Н.И. Леонюк. Физика низких температур, 44 (5), 592 (2018). [A.N. Bludov, Y.O. Savina, M.I. Kobets, V.A. Pashchenko, S.L. Gnatchenko, N.N. Kuzmin,
V.V. Mal'tsev, N.I. Leonyuk. Low Temperature Physics, 44 (5), 453 (2018). DOI: 10.1063/1.5034159]

[45] A. Bludov, Y. Savina, M. Kobets, V. Khrustalyov, V. Savitsky, S. Gnatchenko, T. Zajarniuk, A. Lynnyk, M.U. Gutowska, A. Szewczyk, N. Kuzmin, V. Mal'tsev, N. Leonyuk. J. Magnetism and Magnetic Materials, 512, 167010 (2020). DOI: $10.1016 /$ j.jmmm.2020.167010

[46] Н.Н. Кузьмин, В.В. Мальцев, Е.А. Волкова, Н.И. Леонюк, К.Н. Болдырев, А.Н. Блудов. Неорганические материалы, $\mathbf{5 6}(8), 873$ (2020). [N.N. Kuzmin, V.V. Maltsev, E.A. Volkova, N.I. Leonyuk, K.N. Boldyrev, A.N. Bludov. Inorganic Materials, 56 (8), 828 (2020). DOI: $10.1134 / \mathrm{S} 0020168520080087]$

[47] А.Н. Блудов, Ю.А. Савина, В.А. Пащенко, С.Л. Гнатченко, T. Zajarniuk, A. Lynnyk, M.U. Gutowska, А. Szewczyk, И.В. Колодий, В.В. Мальцев, Н.Н. Кузьмин, Н.И. Леонюк. Физика низких температур, 46 (7), 829 (2020). [A.N. Bludov, Y.O. Savina, V.A. Pashchenko, S.L. Gnatchenko, T. Zajarniuk, A. Lynnyk, M.U. Gutowska, A. Szewczyk, I.V. Kolodiy, V.V. Mal'tsev, N.N. Kuzmin, N.I. Leonyuk. Low Temperature Physics, 46 (7), 697 (2020). DOI: $10.1063 / 10.0001367]$

[48] E.A. Dobretsova, K.N. Boldyrev, M.N. Popova, V.A. Chernyshev, E.Y. Borovikova, V.V. Maltsev, N.I. Leonyuk. J. Physics: Conference Series, 737, 012035 (2016). DOI: $10.1088 / 1742-6596 / 737 / 1 / 012035$

[49] Е.Л. Белоконева, М.А. Симонов, А.В. Пашкова, Т.И. Тимченко, Н.В. Белов. Доклады Академии наук СССР, 255 (4), 854 (1980).

[50] V. Maltsev, E. Janod. J. Crystal Growth, 240 (1-2), 170 (2002). DOI: 10.1016/S0022-0248(02)00856-4

[51] M.N. Popova, T.N. Stanislavchuk, B.Z. Malkin, L.N. Bezmaternykh. J. Physics: Condensed Matter, 24 (19), 196002 (2012). DOI: 10.1088/0953-8984/24/19/196002

[52] Л.Н. Безматерных, С.А. Харламова, В.Л. Темеров. Кристаллография, 49 (5), 944 (2004). [L.N. Bezmaternykh, S.A. Kharlamova, V.L. Temerov. Crystallography Reports, 49 (5), 855 (2004). DOI: 10.1134/1.1803319]

[53] В.Ф. Золин, Л.Г. Коренева Редкоземельный зонд в химии и биологии (Наука, Москва, 1980)

[54] G.G. Chepurco, I.V. Paukov, M.N. Popova, Ja. Zoubkova. Solid State Commun., 79(7), 569 (1991). DOI: 10.1016/0038-1098(91)90911-E

[55] M.N. Popova, S.A. Klimin, R. Troć, Z. Bukowski. Solid State Commun., $\mathbf{1 0 2}(1), \quad 71 \quad$ (1997). DOI: 10.1016/S0038-1098(96)00700-4

[56] N.I. Agladze, G.G. Chepurko, M.N. Popova, E.P. Hlybov. Phys. Lett. A, 133 (4-5), 260 (1988). DOI: $10.1016 / 0375-9601(88) 91028-6$

[57] I.V. Paukov, M.N. Popova, B.V. Mill. Phys. Lett. A, 169 (4), 301 (1992). DOI: 10.1016/0375-9601(92)90463-V

[58] Yu.A. Hadjiiskii, I.V. Paukov, M.N. Popova, B.V. Mill. Phys. Lett. A, 189 (1-2), 109 (1994). DOI: 10.1016/0375-9601(94)90827-3

[59] M.N. Popova, I.V. Paukov, Yu.A. Hadjiiskii, B.V. Mill. Phys. Lett. A, 203 (5-6), 412 (1995). DOI: $10.1016 / 0375-9601(95) 00391-F$

[60] M.N. Popova. J. Magnetism and Magnetic Materials, 321 (7), 716 (2009). DOI: 10.1016/j.jmmm.2008.11.033

[61] M.N. Popova, E.P. Chukalina, T.N. Stanislavchuk, L.N. Bezmaternykh. J. Magnetism and Magnetic Materials, 300 (1), e440 (2006). DOI: 10.1016/j.jmmm.2005.10.187 
[62] M.N. Popova. J. Alloys and Aompounds, 275-277, 142 (1998). DOI: 10.1016/S0925-8388(98)00292-8

[63] K.V. Zakharov, E.A. Zvereva, M.M. Markina, M.I. Stratan, E.S. Kuznetsova, S.F. Dunaev, P.S. Berdonosov, V.A. Dolgikh, A.V. Olenev, S.A. Klimin, L.S. Mazaev, M.A. Kashchenko, Md.A. Ahmed, A. Banerjee, S. Bandyopadhyay, A. Iqbal, B. Rahaman, T. Saha-Dasgupta, A.N. Vasiliev. Phys. Rev. B, 94, 054401 (2016). DOI: 10.1103/PhysRevB.94.054401 